\title{
§ 10 Einstweiliger Rechtsschutz 3: Der Antrag nach § 123 VwGO
}

1 Der gutachterlichen Prüfung des Antrags nach $\S 123$ VwGO liegt die folgende Struktur zugrunde:

A. Zulässigkeit

I. Eröffnung des Verwaltungsrechtswegs (dazu bereits § 1 Rn. 162ff.)

II. Statthafte Antragsart (dazu einführend §1 Rn. 222ff. und ausführlich in diesem $\S$ Rn. 2ff.)

III. Antragsbefugnis (dazu Rn. $11 \mathrm{ff}$.)

IV. Antragsfrist (dazu Rn. 16)

V. Beteiligte (dazu Rn. $17 \mathrm{ff}$.)

VI. Zuständiges Gericht (dazu Rn. 20f.)

VII. Rechtsschutzbedürfnis (dazu Rn. $21 \mathrm{ff}$.)

B. Begründetheit (zur Struktur der Begründetheitsprüfung einführend Rn. $27 \mathrm{ff}$.)

I. Anordnungsgrund (näher dazu Rn. 34)

II. Anordnungsanspruch (näher dazu Rn. $35 \mathrm{ff}$.)

III. Keine Vorwegnahme der Hauptsache (näher dazu Rn. 41ff.)

Näher zum Antrag nach $\S 123$ VwGO im Baurecht Rn. 45 und im Kommunalrecht Rn. $46 \mathrm{ff}$.

\section{Gliederung}

A. Die Statthaftigkeit des Antrag nach $\S 123$ VwGO (Mira Wichmann) — 949

I. Abgrenzung des $\S 123$ VwGO zu $\S \S 80,80$ a VwGO — 950

II. Abgrenzung zu weiteren Formen vorbeugenden Rechtsschutzes — 951

III. Sicherungs- und Regelungsanordnung — 951

B. Weitere Zulässigkeitsvoraussetzungen -953

I. Antragsbefugnis (Hendrik Burbach/Mira Wichmann) — 953

II. (Kein) Vorverfahren (Renana Braun) — 954

III. (Keine) Antragsfrist (Patrick Stockebrandt) - 954

IV. Beteiligte (Carola Creemers) - 955

V. Zuständiges Gericht (Katharina Goldberg) — 955

VI. Rechtsschutzbedürfnis (Dana-Sophia Valentiner/Mira Wichmann) - 955

C. Begründetheit — 957

I. Die Struktur der Begründetheitsprüfung (Mira Wichmann) - 957

1. Glaubhaftmachung 957

2. Anordnungsgrund -958

Ә OpenAccess. (C) 2019 Nikolas Eisentraut, publiziert von De Gruyter. (cc) BY-SA Dieses Werk ist lizenziert unter der Creative Commons Attribution-ShareAlike 4.0. International. 
3. Anordnungsanspruch -959

4. Summarische Rechtsprüfung — 960

5. Verbot der Vorwegnahme und Überschreitung der Hauptsache $-\mathbf{9 6 1}$

6. Literaturhinweise - 962

II. Der Antrag nach § 123 VwGO im Baurecht (Felix Steengrafe) - 963

III. Der Antrag nach § 123 VwGO im Kommunalrecht (Sebastian Piecha) 963

\section{A. Die Statthaftigkeit des Antrag nach § 123 VwGO (Mira Wichmann)}

Wurde der Verwaltungsrechtsweg für eröffnet erkannt (s. ausführlich zur Prü- 2 fung § 1 Rn. 162ff.), so ist im Rahmen der Zulässigkeit als nächstes die statthafte Klage- bzw. Antragsart zu untersuchen. Die Prüfung der statthaften Klage-/Antragsart ist das zentrale Scharnier für die gesamte restliche Klausur. Nach ihr richten sich sowohl die weiteren Zulässigkeitsvoraussetzungen als auch die Struktur der Begründetheitsprüfung. Entsprechend wichtig ist die saubere Prüfung, welche Klage- bzw. Antragsart einschlägig ist (eine erste Übersicht über die Klage- und Antragsarten der VwGO findet sich in $\S 1$ Rn. $222 \mathrm{ff}$.).

Der Bürger muss jedes staatliche Handeln oder Unterlassen, das seine sub- 3 jektiven Rechte beeinträchtigt, praktisch wirksam zur gerichtlichen Überprüfung stellen können. ${ }^{1}$ Dies folgt aus dem Gebot des effektiven Rechtsschutzes, Art. 19 IV 1 GG. „Tatsächlich wirksam“ meint dabei oft „rechtzeitige“ gerichtliche Kontrolle. Die oben dargestellten Klagearten haben jedoch das Manko, dass es lange dauert bis eine Hauptsacheentscheidung über sie getroffen wird. Um zu verhindern, dass diese Entscheidungen für den Kläger zu spät kommen, ihm also ein Rechtsverlust durch Zeitablauf droht, ${ }^{2}$ wurde die Möglichkeit einer schnellen gerichtlichen Entscheidung im Wege des Eilrechtsschutzes getroffen. ${ }^{3}$ Auf diese Weise kann verhindert werden, dass durch den Vollzug einer Verwaltungsmaßnahme vollendete (unabänderbare) Tatsachen geschaffen werden, ohne dass der Betroffene dagegen wirksam Rechtsschutz suchen kann. ${ }^{4}$ Daher bietet neben

1 BT-Drs. III/55, S. 44; BVerfG, Beschl. v. 13.6.1979, Az.: BvR 699/77 = BVerfGE 51, 268 (285f.). 2 Wird ein solcher Rechtsverlust durch Zeitablauf nicht verhindert, kann der Betroffene im Nachhinein mit Hilfe der Fortsetzungsfeststellungsklage gemäß § 113 I 4 VwGO (ggf. analog) Rechtsschutz suchen. Vergleiche hierzu die Ausführungen bei § 4 Rn. $2 \mathrm{ff}$.

3 Buchheister, DVBl 2017, 610 (611); vergleiche zur Entstehungsgeschichte die ausführliche Darstellung von Schoch, in: Schoch/Schneider/Bier, VwGO, 36. EL Februar 2019, § 123 Rn. 4-8.

4 Hufen, Verwaltungsprozessrecht, 11. Aufl. 2019, § 31 Rn. 2; Schoch, in: Schoch/Schneider/Bier, VwGO, 36. EL Februar 2019, § 123 Rn. 3. 
$\S 80$ V VwGO auch § 123 VwGO die Möglichkeit des Eilrechtsschutzes. ${ }^{5}$ Man spricht daher auch von dem zweispurigen System des vorläufigen Rechtsschutzes ( $\$ \S 80$, 80a VwGO einerseits, $§ 123$ VwGO andererseits). ${ }^{6}$ Eilrechtsschutz meint dabei nur eine vorübergehende Sicherung der Rechte der Beteiligten bis eine Entscheidung in der Hauptsache ergeht. ${ }^{7}$

4 Die Statthaftigkeit des Antrags richtet sich wie bei allen anderen Klage- und Antragsarten nach dem Begehren des Antragstellers, §§ 88 VwGO. Da § 88 VwGO seinem Wortlaut nach nur für Klagen Anwendung findet, § 123 VwGO jedoch ein Antragsverfahren ist, zitiert man $\S 122$ I VwGO, der ausdrücklich auf $\S 88$ VwGO verweist, im einstweiligen Rechtsschutz stets mit. In Klausuren wird sich der Fall in der Regel so gestalten, dass der Betroffene möglichst schnell gerichtlich Rechtsschutz in Anspruch nehmen möchte. Dies lässt zunächst allgemein auf den Eilrechtsschutz schließen. Innerhalb der Statthaftigkeit ist sodann zwischen den unterschiedlichen Arten des Eilrechtsschutzes abzugrenzen.

\section{Abgrenzung des $\S 123$ VwG0 zu $\S \S 80,80 a$ VwGO}

5 Die Anträge nach $\S 80,80 a$ und 123 VwGO sind exklusiv, d.h. sie schließen sich gegenseitig aus. Das geht aus $\S 123$ V VwGO hervor, denn danach gelten die Absätze 1 bis 3 des $\S 123$ VwGO nicht für die Fälle der $\S \S 80,80 a$ VwGO. ${ }^{8}$ Der Antrag nach $\S 123$ VwGO ist als Auffangtatbestand somit zum Antrag nach $\S \S 80$, 80a VwGO subsidiär. ${ }^{9}$ Ein Antrag nach $\S 123$ VwGO ist also immer dann statthaft, wenn nicht $\S \S 80$, 80a VwGO statthaft sind. Negativ formuliert, darf also nicht ein statthafter Anfechtungswiderspruch oder eine statthafte Anfechtungsklage zum begehrten Rechtsschutzziel führen. ${ }^{10}$ Positiv formuliert, liegt demnach die Statthaftigkeit des Antrags nach $\S 123$ I VwGO vor, wenn der Antragsteller ein Vorgehen im einstweiligen Rechtsschutz begehrt, das auf Erlass eines Verwaltungsakts

5 Auch der Normenkontrollantrag nach §47 I sieht in Absatz 6 VwGO die Möglichkeit des einstweiligen Rechtsschutzes vor.

6 Schoch, in: Schoch/Schneider/Bier, VwGO, 36. EL Februar 2019, § 123 Rn. 1.

7 Hufen, Verwaltungsprozessrecht, 11. Aufl. 2019, § 31 Rn. 6. In der Praxis ist jedoch zu beobachten, dass der Rechtsstreit in der Hauptsache gar nicht zu Ende geführt wird, sondern sich die Beteiligten mit einer vorläufigen Entscheidung zu Frieden geben. Zur Abgrenzung vom vorbeugenden Rechtschutz s. unten die Ausführungen zum Rechtschutzbedürfnis in diesem § Rn. $22 \mathrm{ff}$. sowie Rn. 6.

8 In § 123 V VwGO ist die sog. „Subsidiaritätsklausel“ enthalten. Schoch, in: Schoch/Schneider/ Bier, VwGO, 36. EL Februar 2019, § 123 Rn. 1.

9 Schoch, in: Schoch/Schneider/Bier, VwGO, 36. EL Februar 2019, § 123 Rn. 1.

$10 \mathrm{~S}$. dazu die Ausführungen oben zu $\S 80 \mathrm{~V}$ VwGO in $\S 8 \mathrm{Rn} .2 \mathrm{ff}$.

\section{Mira Wichmann}


gerichtet ist oder wenn ein faktisches Verwaltungshandeln angestrebt oder abgewehrt werden soll. ${ }^{11}$ Somit sind die Fälle der Verpflichtungs-, allgemeinen Leistungs- oder Feststellungsklage erfasst. ${ }^{12} \S 123$ VwGO greift also immer dann, wenn in der Hauptsache keine Anfechtungsklage, sondern eine der anderen verwaltungsrechtlichen Klagearten statthaft ist. ${ }^{13}$

\section{Abgrenzung zu weiteren Formen vorbeugenden Rechtsschutzes}

Große Ähnlichkeit zum Eilrechtsschutz besteht in Fällen des vorbeugenden 6 Rechtschutzes, z. B. bei der vorbeugenden Feststellungsklage und der vorbeugenden Unterlassungsklage. Vorbeugender Rechtsschutz kann ebenfalls zur Anwendung kommen, wenn ein bevorstehendes Rechtsverhältnis streitig ist, der Erlass eines Verwaltungsakts bevorsteht ${ }^{14}$ oder eine behördliche Maßnahme droht, die irreversible Tatsachen zur Folge hätte und dadurch nicht wiedergutzumachende Nachteile zu erwarten sind. ${ }^{15}$ Daher ist gegebenenfalls in einer Klausur herauszuarbeiten, warum der vorläufige Rechtsschutz und nicht der vorbeugende Rechtsschutz statthaft ist. Während der vorbeugende Rechtsschutz im Hauptsacheverfahren statthaft ist, ist der vorläufige Rechtsschutz Teil des Eilrechtsschutzes. Abzugrenzen sind diese beiden Arten somit über die Eilbedürftigkeit. Bei $\S 123$ VwGO ist diese Voraussetzung im Anordnungsgrund zu prüfen. Auch, wenn in der Hauptsache der vorbeugende Rechtsschutz statthaft ist, kann im Eilrechtsschutz ein Antrag nach $§ 123$ VwGO statthaft sein.

\section{Sicherungs- und Regelungsanordnung}

§123 I VwGO enthält zwei Anordnungsarten zwischen denen innerhalb der 7 Statthaftigkeit zu differenzieren ist: Oftmals wird auf diese Differenzierung verzichtet, da sie weniger eindeutig ist als die Darstellung suggeriert. In der Klausur sollte dennoch kurz dazu Stellung genommen werden.

Satz 1 lautet: Auf Antrag kann das Gericht, auch schon vor Klageerhebung, eine einstweilige Anordnung in Bezug auf den Streitgegenstand treffen, wenn die Gefahr besteht, dass durch eine Veränderung des bestehenden Zustands die

11 Kuhla, in: Posser/Wolff, VwGO, 50. Ed., Stand: 1.7.2019, § 123, vor A.

12 Schenke, Verwaltungsprozessrecht, 16. Aufl. 2019, § 25 Rn. 1025.

13 Hufen, Verwaltungsprozessrecht, 11. Aufl. 2019, § 31 Rn. 7.

14 Schoch, in: Schoch/Schneider/Bier, VwGO, 36. EL Februar 2019, § 123 Rn. 45.

15 Schoch, in: Schoch/Schneider/Bier, VwGO, 36. EL Februar 2019, § 123 Rn. 46.

Mira Wichmann 
Verwirklichung eines Rechts des Antragstellers vereitelt oder wesentlich erschwert werden könnte.

Satz 2 lautet: Einstweilige Anordnungen sind auch zur Regelung eines vorläufigen Zustands in Bezug auf ein streitiges Rechtsverhältnis zulässig, wenn diese Regelung, vor allem bei dauernden Rechtsverhältnissen, um wesentliche Nachteile abzuwenden oder drohende Gewalt zu verhindern oder aus anderen Gründen nötig erscheint.

8 Satz 1 wird Sicherungsanordnung genannt. Sie ist statthaft, wenn die Gefahr besteht, dass durch eine Veränderung des bestehenden Zustandes die Verwirklichung eines Rechts des Antragsstellers vereitelt oder wesentlich erschwert werden könnte. ${ }^{16}$ Die Sicherungsanordnung dient somit der Wahrung des tatsächlichen oder rechtlichen Zustandes, mithin dem Erhalt des status quo. ${ }^{17}$ Häufig werden hier Abwehransprüche relevant. Der typischste Anwendungsfall eines Abwehranspruchs ist die Sicherung von Unterlassungsansprüchen in Form der Konkurrentenklage. ${ }^{18}$ Diese würden in der Hauptsache durch Leistungsklage in Gestalt der Abwehrklage geltend gemacht werden. ${ }^{19}$ Insoweit dient die Sicherungsanordnung (§ 123 I 1) als funktionales Äquivalent zur aufschiebenden Wirkung der Gewährleistung eines wirksamen vorläufigen Rechtsschutzes. ${ }^{20}$

Beispiele für die Statthaftigkeit der Sicherungsanordnung: Antrag auf Aufrechterhaltung der Zugehörigkeit eines Schülers zur Schule; Antrag eines Behördenmitarbeiters gegen eine Dienstanordnung des Behördenleiters; Antrag auf ein vorläufiges Verbot der Ernennung/Beförderung eines Konkurrenten; Antrag auf ein Verbot der Versteigerung einer Fundsache; Antrag auf ein vorläufiges Verbot der Auszahlung einer Subvention; Antrag auf Unterlassung ehrverletzender oder geschäftsschädigender Äußerungen; Antrag auf Unterlassung der Durchführung öffentlicher Maßnahmen, z.B. der Bauarbeiten für öffentliche Vorhaben.

9 Satz 2 enthält die sogenannte Regelungsanordnung. Im Gegensatz zu Satz 1 geht es hier nicht um die Bewahrung eines bestehenden Zustandes, sondern um eine vorläufige Veränderung des Zustandes. ${ }^{21}$ Die Regelung eines vorläufigen Zustands in Bezug auf ein streitiges Rechtsverhältnis kann begehrt werden, um wesentliche Nachteile abzuwenden oder drohende Gefahren zu verhindern. ${ }^{22}$ Einfach ge-

16 Hummel, JuS 2011, 502.

17 Schoch, in: Schoch/Schneider/Bier, VwGO, 36. EL Februar 2019, § 123 Rn. 50, 52; Hufen, Verwaltungsprozessrecht, 11. Aufl. 2019, S. 525 Rn. 14.

18 Schoch, in: Schoch/Schneider/Bier, VwGO, 36. EL Februar 2019, § 123 Rn. 53, 40.

19 Schmidt, JA 2002, 885 (887).

20 Schoch, in: Schoch/Schneider/Bier, VwGO, 36. EL Februar 2019, § 123 Rn. 12.

21 Hufen, Verwaltungsprozessrecht, 11. Aufl. 2019, S. 525 Rn. 15.

22 Hummel, JuS 2011, 502.

Mira Wichmann 
sprochen, ist die Regelungsanordnung statthaft, wenn die Einräumung oder Erweiterung einer Rechtsposition begehrt wird, ${ }^{23}$ also bei Verpflichtungs- und Feststellungsbegehren oder der Leistungsklage als Vornahmeklage ${ }^{24}$. Das streitige Rechtsverhältnis wird nämlich auch in diesem Zusammenhang wie bei §43 I VwGO verstanden.

Beispiele für die Statthaftigkeit der Regelungsanordnung: Antrag eines Veranstalters gegen die Stadt Hamburg auf Duldung der Durchführung eines Protestcamps gegen G2O25; Antrag auf Zulassung eines Standes auf einem Weihnachts-/oder Wochenmarkt; Antrag auf Zulassung zu einer Prüfung, über den Fortgang einer Prüfung, über die (vorläufige) Neubewertung einer Prüfungsarbeit oder über die (vorläufige) Erteilung eines Zeugnisses oder einer Bescheinigung $^{26}$; Antrag auf (Wieder-)Erteilung der Fahrerlaubnis ${ }^{27}$; Antrag auf Zuweisung eines Schulplatzes an einer bestimmten Schule ${ }^{28}$; Antrag gegen die Aufrechterhaltung von amtlichen Publikumsinformationen, die vorläufige Zulassung zur Ersten Juristischen Staatsprüfung ${ }^{29}$.

\section{B. Weitere Zulässigkeitsvoraussetzungen}

Nach der Prüfung der Eröffnung des Verwaltungsrechtswegs und der Prüfung 10 der statthaften Antragsart sind im Falle des Antrags nach $\S 123$ VwGO regelmäßig die folgenden weiteren Zulässigkeitsvoraussetzungen anzusprechen, wobei es auch hierbei auf problembewusstes Arbeiten ankommt (dazu §1 Rn. 52, 123).

\section{Antragsbefugnis (Hendrik Burbach/Mira Wichmann)}

Auch für die Antragsbefugnis im Verfahren nach $\S 123$ VwGO gilt § 42 II VwGO 11 analog. Der Antragsteller muss ein Recht geltend machen können, das ihm zukommt und das möglicherweise verletzt oder gefährdet ist. ${ }^{30}$

23 Schoch, in: Schoch/Schneider/Bier, VwGO, 36. EL Februar 2019, § 123 Rn. 50.

24 Schmidt, JA 2002, 885 (888).

25 Vgl.VG Hamburg, Beschl.v. 7.6.2017, Az.: 19 E 5697/17 = BeckRS 2017, 116503; dazu Hartmann, NVwZ 2018, 200 (202).

26 Zimmerling/Brehm, NVwZ 2004, 651.

27 VGH München, Beschl. v. 23.6.2016, Az.: 11 CE 16.851, 11 C 16.850; s. dazu auch die Besprechung mit weiteren Beispielen aus dem Verwaltungsverkehrsrecht bei Weber, DAR 2018, 172.

28 Grünberg, LKV 2014, 433.

29 SächsOVG, Beschl. v. 6.0.1997, Az.: 4 S 135/97 = DtZ 1997, 235 (236).

30 Wollenschläger, in: Gärditz, VwGO, 2. Aufl. 2019, § 123 Rn. 85; Hufen, Verwaltungsprozessrecht, 11. Aufl. 2019, §33 Rn. 9. 
Die Möglichkeit der Verletzung in eigenen subjektiven Rechten bedeutet bei einem Antrag nach $\S 123$ VwGO, dass die Möglichkeit eines Anordnungsanspruches und eines Anordnungsgrundes von dem Antragssteller darzulegen sind. ${ }^{31}$

13 Begehrt der Antragsteller eine Sicherungsanordnung, muss aus dem Antrag ersichtlich sein, dass die Gefahr besteht, durch die Veränderung des bestehenden Zustandes die Verwirklichung eines Rechts des Antragstellers vereitelt oder wesentlich erschwert werden könnte. ${ }^{32}$ Ist der Antrag auf eine Regelungsanordnung gerichtet, muss der Antragsteller darlegen, dass diese zur Abwendung wesentlicher Nachteile oder einer drohenden Gefahr erforderlich ist. ${ }^{33}$

14 Formulierungsvorschlag: „Der Antragsteller müsste zudem auch antragsbefugt nach § 42 II VwGO analog sein. Dies setzt voraus, dass er einen Anordnungsanspruch und Anordnungsgrund geltend machen kann (...).“

\section{II. (Kein) Vorverfahren (Renana Braun)}

15 Im Rahmen der Zulässigkeit eines Antrags nach § 123 VwGO ist die erfolglose Durchführung eines Vorverfahrens keine Sachentscheidungsvoraussetzung und daher in einer Klausur nicht zu thematisieren.

\section{III. (Keine) Antragsfrist (Patrick Stockebrandt)}

16 Grundsätzlich ist beim Antrag nach $\S 123$ VwGO keine Frist zu beachten. ${ }^{34}$ Ein Antrag ist also auch schon vor dem Hauptsacheverfahren bzw. während des Widerspruchsverfahrens möglich, sofern das Rechtschutzbedürfnis besteht. Jedoch können spezialgesetzliche Regelungen, wie z.B. im Asylrecht, eine Frist ausnahmsweise anordnen. ${ }^{35}$ Im Hinblick auf die Fristberechnung (s. § 2 Rn. 361 ff.) kann auf die Ausführungen im Rahmen der Anfechtungsklage verwiesen werden.

Weiterführende Literaturhinweise finden sich in $\S 2$ Rn. 402.

31 Schoch, in: Schoch/Schneider/Bier, VwGO, 36. EL Februar 2019, § 123 Rn. 107.

32 Hufen, Verwaltungsprozessrecht, 11. Aufl. 2019, § 33 Rn. 9.

33 Hufen, Verwaltungsprozessrecht, 11. Aufl. 2019, § 33 Rn. 9.

34 Schenke, in: Kopp/Schenke, VwGO, 25. Aufl. 2019, § 123 Rn. 21. Zur besonderen Situation bei unterlegenen Bewerbern um eine Beamtenstelle s. Schenke, in: Kopp/Schenke, VwGO, 25. Aufl. 2019, § 123 Rn. 21.

35 Befristet ist der Antrag auf Gewährung vorläufigen Rechtsschutzes i.V.m. einem Asylantrag gemäß § 18a IV 1 AsylG, s. Schenke, in: Kopp/Schenke, VwGO, 25. Aufl. 2019, § 123 Rn. 21. 


\section{Beteiligte (Carola Creemers)}

Die Ausführungen zur Beteiligungs- und Prozessfähigkeit im Rahmen der 17 Anfechtungsklage gelten entsprechend für den Antrag nach § 123 VwGO (s. ausführlich § 2 Rn. $403 \mathrm{ff}$.).

Der richtige Antragsgegner bestimmt sich im einstweiligen Rechtsschutz 18 nach dem richtigen Beklagten in der Hauptsache. Demzufolge kann nur dann auf $\S 78$ VwGO analog abgestellt werden, wenn es sich in der Hauptsache um eine Verpflichtungsklage handelt. Im Übrigen bestimmt sich der richtige Antragsgegner nach dem allgemeinen Rechtsträgerprinzip (s. dazu § 5 Rn. 37 a.E).

Auch im einstweiligen Rechtsschutzverfahren nach $\S 123$ VwGO sind die 19 Beiladung nach $\S 65 \mathrm{VwGO}^{36}$ und die subjektive Antragshäufung nach $\S 64$ $\mathrm{VwGO}^{37}$ analog möglich, sodass auf die Ausführungen zur Anfechtungsklage verwiesen werden kann (vgl. § 2 Rn. 445 ff., 455 ff.), wobei diese im Verfahren nach $\S 123$ VwGO relativ selten sind.

\section{Zuständiges Gericht (Katharina Goldberg)}

Das zuständige Gericht im einstweiligen Rechtsschutz nach $\S 123$ VwGO ist gem. 20 $\S 123$ II 1, 2 VwGO das Gericht der Hauptsache. Dies ist nach § 123 II 2 VwGO grundsätzlich das Gericht des ersten Rechtszugs. Wenn die Hauptsache im Berufungsverfahren anhängig ist, ist hingegen das Berufungsgericht zuständig (s. zur Zuständigkeit in den jeweiligen Hauptsacheverfahren $\S 3$ Rn. 38ff. (Verpflichtungsklage), § 5 Rn. $40 \mathrm{ff}$. (allgemeine Leistungsklage) und §6 Rn. $89 \mathrm{ff}$. (Feststellungsklage)).

Formulierungsvorschlag: „Das Gericht der Hauptsache ist gem. $\S 123$ II 1, 221 VwGO im einstweiligen Rechtsschutz zuständig. Dessen Zuständigkeit ergibt sich sachlich aus $\S 45$ VwGO und örtlich aus $\S 52$ VwGO.“

\section{Rechtsschutzbedürfnis (Dana-Sophia Valentiner/Mira Wichmann)}

Auch ein Antrag nach $\S 123$ VwGO erfordert, dass die Antragstellerin rechts- 22 schutzbedürftig ist (s. einleitend zum Erfordernis des Rechtsschutzbedürfnisses bereits $§ 2 \mathrm{Rn} .477 \mathrm{ff}$.).

36 Schenke, in: Kopp/Schenke, VwGO, 25. Aufl. 2019, § 65 Rn. 3, § 123 Rn. 1.

37 Kintz, in: Posser/Wolff, VwGO, 50. Ed., Stand: 1.4.2019, § 64 Rn. 2. 
Grundsätzlich ist für das Rechtsschutzbedürfnis erforderlich, dass kein einfacherer, schnellerer und gleich effektiverer Rechtsschutz in Betracht kommt. Daher ist für einen Antrag nach $\S 123$ VwGO grundsätzlich ein - zumindest konkludenter - Antrag bei der zuständigen Behörde zu stellen. ${ }^{38}$ Denn nur so ist gewährleistet, dass die Behörde überhaupt Kenntnis davon erlangt, dass der Betroffene sich gegen Verwaltungsmaßnahmen wenden möchte. Das behördliche Verwaltungsverfahren kann nämlich einen einfacheren und gleich effektiven Rechtsschutz zum gerichtlichen Verfahren leisten.

Ausnahmen vom Antragserfordernis gelten bei besonderer Eilbedürftigkeit der Sache und einer geringen Wahrscheinlichkeit, dass der Antrag rechtzeitig positiv von der Behörde entschieden wird. ${ }^{39}$

Deutet das Verhalten der Antagstellerin, etwa durch zögerliches Betreiben der Hauptsache, darauf hin, dass sie die Sache nicht für eilbedürftig hält oder hat sie bereits einen vorläufig vollstreckbaren Titel erwirkt, kann dies der Annahme des Rechtschutzbedürfnisses entgegen stehen. ${ }^{40}$

Das Rechtsschutzbedürfnis einer antragstellenden Behörde fehlt, wenn sie die begehrte Regelung durch eigenes Verwaltungshandeln herbeiführen kann. ${ }^{41}$

In Fällen des vorbeugenden Rechtsschutzes (Unterlassungsklage, Feststellungsklage) kommt dem Rechtsschutzbedürfnis eine besondere Relevanz zu. Denn grundsätzlich ist es der Bürgerin vor Beschreiten des gerichtlichen Rechtsweges zumutbar, eine behördliche Maßnahme abzuwarten. Dies folgt u.a. aus dem Gewaltenteilungsprinzip des Art. 20 II GG, der es gerade vermeiden soll, die Entscheidungsfreiheit der Verwaltung durch richterliche Anordnung einzuengen. ${ }^{42}$ Daher ist in einem solchen Fall des vorläufig vorbeugenden Rechtsschutzes ein besonderes Interesse an der vorbeugenden Gewährung von Rechtsschutz zu begründen..$^{43}$ Dabei muss bei der Prüfung des Rechtschutzbedürfnisses dargelegt werden, dass der Antragstellerin eine irreversible Rechtsbeeinträchtigung droht, die es rechtfertigt schon vorläufig im Wege der einstweiligen Anordnung den vorbeugenden Rechtsschutz zu gewähren. ${ }^{44}$

38 Schoch, in: Schoch/Schneider/Bier, VwGO, 36. EL Februar 2019, § 123 Rn. 121b; Gersdorf, Verwaltungsprozessrecht, 6. Aufl. 2019, Rn. 213.

39 W.-R. Schenke, in: Kopp/Schenke, VwGO, 25. Aufl. 2019, § 123 Rn. 22; Puttler, in: Sodan/Ziekow, VwGO, 5. Aufl. 2018, § 123 Rn. 70.

40 Hufen, Verwaltungsprozessrecht, 11. Aufl. 2019, S. 525 Rn. 10.

41 OVG Hamburg, Beschl. v. 22.10.1988, Az.: Bs. I 195/88 = NJW 1989, 605; Schoch, in: Schoch/ Schneider/Bier, VwGO, 36. EL Februar 2019, § 123 Rn. 121a.

42 M.w.N. Kuhla, in: Posser/Wolff, VwGO, 50. Ed., Stand: 1.7.2019, § 123, Rn. 43.

43 Puttler, in: Sodan/Ziekow, VwGO, 5. Aufl. 2018, § 123 Rn. 71.

44 Puttler, in: Sodan/Ziekow, VwGO, 5. Aufl. 2018, § 123 Rn. 71. 


\section{Begründetheit}

Ist der Antrag nach $\S 123$ VwGO zulässig, ist in einem zweiten Schritt zu prüfen, ob 26 er auch begründet ist.

\section{Die Struktur der Begründetheitsprüfung (Mira Wichmann)}

In der Klausur wäre der Obersatz zur Prüfung der Begründetheit eines Antrages 27 nach § 123 I VwGO wie folgt zu bilden. Dabei muss sich zwischen $\S 123$ I 1 und 2 VwGO entschieden werden:

„Der Antrag auf Erlass einer einstweiligen Anordnung ist begründet, soweit der Antragssteller gemäß $\S 123$ I 1 oder 2, III VwGO i.V.m. \$§ 920 II, 294 I ZPO einen Anordnungsanspruch und Anordnungsgrund glaubhaft gemacht hat und keine unzulässige Vorwegnahme der Hauptsache vorliegt.“

\section{Glaubhaftmachung}

Anders als bei den verwaltungsaktbezogenen Klagearten kommt es bei einem 28 Antrag nach § 123 I VwGO nicht auf eine Rechtsverletzung des Antragsstellers an; es genügt für die Begründetheit des Antrags, dass der Anordnungsanspruch, genauer der zugrunde liegende Sachverhalt, glaubhaft gemacht wurde. Dabei sind die Nachweise von Tatsachen vom Antragsteller glaubhaft zu machen, die einen Anordnungsanspruch begründen. Glaubhaftmachung ist gegenüber dem Vollbeweis eine reduzierte Form des Beweises. Es bedarf der Glaubhaftmachung nur, wenn die Tatsachen streitig sind..$^{45}$

In der Klausur des erstens Examens wird dies regelmäßig nicht zu prüfen 29 sein, da der Sachverhalt die vollständig ermittelten Tatsachen enthält und keine Beweiserhebung vorzunehmen ist.

Die von $\S 123$ III VwGO vorausgesetzte Glaubhaftmachung bezieht sich dabei 30 auf die § 294 I ZPO i.V.m. § 920 II ZPO. Sie meint eine bestimmte Art der Beweisführung, die darauf gerichtet ist, den Richter zu überzeugen, dass er die behauptete Tatsache für wahrscheinlicher hält als das Gegenteil. ${ }^{46}$ Die Glaubhaftmachung kann durch alle Beweismittel des § 294 I ZPO erfolgen, die auch an Eides statt zugelassen werden. ${ }^{47}$

45 Heetkamp/Stadermann, JA 2015, 933 (936).

46 Huber, in: Musielak/Voit, ZPO, 16. Aufl. 2019, § 294 Rn. 3.

47 Hummel, JuS 2011, 502 (503). 
Hier liegt auch ein wesentlicher Unterschied zum Hauptsacheverfahren. Denn dort darf der Richter eine Tatsache nur dann zum Gegenstand seiner rechtlichen Beurteilung machen, wenn er am Ende der Beweisaufnahme die Überzeugung gewonnen hat, dass die behauptete Tatsache wahr ist. Beim vorläufigen Rechtsschutz reicht hingegen schon die überwiegende Wahrscheinlichkeit einer Tatsache aus. ${ }^{48}$

32 Dieses Vorgehen im Eilrechtsschutz steht auch nicht dem im Verwaltungsrecht geltenden Amtsermittlungsgrundsatz entgegen. Die tatsächlichen Voraussetzungen des Anordnungsanspruchs und des Anordnungsgrundes sind auch hier von Amts wegen zu ermitteln. Sie können insgesamt aber als glaubhaft gemacht angesehen werden ,...., wenn das Gericht auf Grund der vom Antragsteller glaubhaft gemachten und/oder auf Grund der vom Gericht ermittelten bzw. als hinreichend wahrscheinlich bejahten Tatsachen den Anordnungsanspruch für aussichtsreich und die behauptete Gefährdung für wahrscheinlich erachtet“. ${ }^{49}$

Demnach ist ein Anordnungsantrag begründet, wenn das Gericht auf Grund einer hinreichenden Tatsachenbasis, die entweder vom Antragsteller glaubhaft gemacht (§ 123 III VwGO i.V.m. § 920 II, § 294 I ZPO) oder im Wege der Amtsermittlung ( $88 \mathrm{VwGO}$ ) festgestellt wurde, einen Anordnungsanspruch und einen Anordnungsgrund bejaht..$^{50}$

\section{Anordnungsgrund}

34 Zunächst müsste ein Anordnungsgrund vorliegen. Der Anordnungsgrund liegt vor, wenn dem Antragsteller ein Abwarten der gerichtlichen Entscheidung in der Hauptsache nicht zugemutet werden kann. ${ }^{51}$ Innerhalb des Anordnungsgrundes muss also vom Antragsteller dargelegt werden, dass bereits im vorläufigen und nicht erst im Hauptsacheverfahren Rechtsschutz gewährleistet werden muss. ${ }^{52}$ Man spricht daher von einer besonderen Eilbedürftigkeit oder Dringlichkeit. ${ }^{53}$ $\mathrm{Zu}$ unterscheiden sind auch hier wieder die zwei Anordnungsarten (s. o.). Bei

48 Kuhla, in: Posser/Wolff, VwGO, 50. Ed., Stand: 1.7.2019, § 123 Rn. $61 \mathrm{f}$.

49 Schoch, in: Schoch/Schneider/Bier, VwGO, 36. EL Februar 2019, § 123 Rn. 95a und Heinze, NZA 1984, 305 (307).

50 Schoch, in: Schoch/Schneider/Bier, VwGO, 36. EL Februar 2019, § 123 Rn. 62; Bostedt, in: Fehling/Kastner/Störmer, Verwaltungsrecht, 4. Aufl. 2016, § 123 Rn. 68.

51 Kuhla, in: Posser/Wolff, VwGO, 50. Ed., Stand: 1.7.2019, § 123, Rn. 122, 126.

52 Schoch, in: Schoch/Schneider/Bier, VwGO, 36. EL Februar 2019, § 123 Rn. 76.

53 Hufen, Verwaltungsprozessrecht, 11. Aufl. 2019, § 33 Rn. 16; Schenke, Verwaltungsprozessrecht, 16. Aufl. 2019, Rn. 1032; Schoch, in: Schoch/Schneider/Bier, VwGO, 36. EL Februar 2019, $\S 123$ Rn. 77a. 
einer Sicherungsanordnung (§ 123 I 1 VwGO) muss der Antragssteller glaubhaft machen, dass eine „Gefahr besteht, dass durch eine Veränderung des bestehenden Zustandes die Verwirklichung eines Rechts des Antragstellers vereitelt oder wesentlich erschwert werden könnte“. Diese Gefahr der Beeinträchtigung muss auch unmittelbar bevorstehen, sodass die dadurch entstehenden Beeinträchtigungen erwartbar sind. ${ }^{54}$ Bei der Regelungsanordnung (§ 123 I 2 VwGO) besteht Eilbedürftigkeit, vor allem bei dauernden Rechtsverhältnissen, um wesentliche Nachteile abzuwenden oder drohende Gewalt zu verhindern oder wenn es aus anderen Gründen nötig erscheint.

Formulierungsvorschlag: „Es müsste eine besondere Eilbedürftigkeit für den Erlass der einstweiligen Anordnung vorliegen. Für die Fälle der Sicherungsanordnung liegt eine solche besondere Eilbedürftigkeit vor, wenn infolge der drohenden Veränderung die Gefahr besteht, dass das betroffene Recht vereitelt oder seine Durchsetzung erschwert wird. Für eine Regelungsanordnung ist die besondere Eilbedürftigkeit anzunehmen, wenn wesentliche Nachteile oder Gefahren drohen oder wenn es aus anderen Gründen nötig erscheint.“

\section{Anordnungsanspruch}

Weitere Voraussetzung für einen begründeten Antrag nach § 123 I 1 oder 2 VwGO 35 und in Klausuren meist der Kern der Prüfung ist der Anordnungsanspruch. Unter Anordnungsanspruch ist eine klagbare Rechtsposition $\mathrm{zu}$ verstehen, die der Antragsteller als Kläger in dem Hauptsacheverfahren geltend macht. ${ }^{55}$ Es ist somit gerade nicht ein Anspruch auf Erlass der einstweiligen Anordnung selbst. ${ }^{56}$ Diese klagbare Rechtsposition wird auch als materiell-rechtliche Anspruch auf Vornahme der geltend gemachten Handlung bezeichnet. Dies erfordert an dieser Stelle jedoch sowohl die formellen als auch materiellen Anspruchsvoraussetzungen. In einem Fall der Sicherungsanordnung kommt es hier gemäß § 123 I 1 VwGO auf die Sicherung eines subjektiven öffentlichen Rechts an. Bei der Regelungsanordnung kommt es hingegen gemäß § 123 I 2 VwGO auf die Klärung eines streitigen Rechtsverhältnisses an. In beiden Fällen geht es jedoch um eine öffentlich rechtliche Norm, die das Verhältnis von Personen untereinander oder zu Sachen regeln. ${ }^{57}$

54 Gersdorf, Verwaltungsprozessrecht, 6. Aufl. 2019, Rn. 214; Schoch, in: Schoch/Schneider/Bier, VwGO, 36. EL Februar 2019, § 123 Rn. 77a.

55 Puttler, in: Sodan/Ziekow, VwGO, 5. Aufl.2018, § 123 Rn. 77.

56 Puttler, in: Sodan/Ziekow, VwGO, 5. Aufl.2018, § 123 Rn. 77.

57 Hummel, JuS 2011, 502 (503).

Mira Wichmann 
Achtung! Im Gegensatz zum Antrag nach $\S \S 80$, 80a VwGO wird hier keine Interessenabwägung vorgenommen. ${ }^{58}$ Es erfolgt jedoch genau wie bei $\S \S 80,80 \mathrm{a}$ VwGO eine Orientierung an den Erfolgsaussichten des Rechtsbehelfs in der Hauptsache $^{59}$, also eine Bewertung der materiellen Rechtslage. ${ }^{60}$

Während bei Anfechtungs-, Verpflichtungs- und Fortsetzungsfeststellungsklage die materiellen Voraussetzungen der streitentscheidenden Norm bei dem Prüfungspunkt „materielle Rechtmäßigkeit des Verwaltungsakts/materielle Anspruchsvoraussetzungen“ zu prüfen sind, werden diese materiellen und die formellen Voraussetzungen bei der einstweiligen Anordnung nach § 123 I VwGO im Rahmen des Anordnungsanspruches geprüft. ${ }^{61}$ Es handelt sich somit bei dem Anordnungsanspruch um den jeweiligen Anspruch, der sich gegen ein oder auf ein behördliches Handeln richtet, wobei hier wieder zwischen den zwei Arten Sicherungs- und Regelungsanspruch zu unterscheiden ist. ${ }^{62}$

38 Abstraktes Formulierungsbeispiel zur Prüfung eines Anordnungsanspruches: „Es müsste ein Anordnungsanspruch vorliegen. Anordnungsanspruch meint eine klagbare Rechtsposition des Antragstellers, die sich aus einem materiellen Anspruch ergeben kann. Im Falle der Sicherungsanordnung muss sich diese Rechtsposition gegen ein behördliches Handeln richten./ Im Falle der Regelungsanordnung muss sich diese Rechtsposition auf die Vornahme der geltend gemachten Handlung beziehen.

Ein solcher Anspruch könnte sich hier aus $\S$... ergeben. Ausweislich seines Wortlautes vermittelt diese Norm eine einklagbare Rechtsposition in Form eines subjektiven-öffentlichen Rechts, sodass sie eine taugliche Anspruchsgrundlage darstellt.

Die formellen und materiellen Anspruchsvoraussetzungen müssten für das Bestehen des Anordnungsanspruches gegeben sein. (...)“

\section{Summarische Rechtsprüfung}

39 Zwar unterliegt der Antrag nach $\S 123$ I VwGO wie die Anträge nach $\S \S 80$, 80a VwGO der summarischen Rechtsprüfung, allerdings bedeutet dies nicht, dass die

58 Schoch, in: Schoch/Schneider/Bier, VwGO, 36. EL Februar 2019, § 123 Rn. 65.

59 Schenke, Verwaltungsprozessrecht, 16. Aufl. 2019, § 25 Rn. 1032; Schoch, in: Schoch/Schneider/Bier, VwGO, 36. EL Februar 2019, § 123 Rn. 69.

60 Gersdorf, Verwaltungsprozessrecht, 6. Aufl. 2019, Rn. 214; Hufen, Verwaltungsprozessrecht, 11. Aufl. 2019, § 33 Rn. 16.

61 Schaks/Friedrich, JuS 2018, 954 (957).

62 Gersdorf, Verwaltungsprozessrecht, 6. Aufl. 2019, Rn. 216. 
auftretenden Rechtsfragen unbeantwortet bleiben können. Vielmehr bezieht sich dies nur auf die Sachverhaltsfeststellungen bei besonders eilbedürftigen Entscheidungen, die nicht erschöpfend vorgenommen werden können. ${ }^{63}$ Die Frage spielt daher in Klausuren regelmäßig keine Rolle.

Beachte: In Klausursituation ist also die Prüfung grundsätzlich genauso $\mathbf{4 0}$ ausführlich vorzunehmen wie bei einem Hauptsacherechtsbehelf.

\section{Verbot der Vorwegnahme und Überschreitung der Hauptsache}

Bei § 123 VwGO gilt das von der Rechtsprechung entwickelte Verbot der Vorweg- 41 nahme der Hauptsache. ${ }^{64}$ Eine endgültige Vorwegnahme der Hauptsache meint, dass dem Antragsteller bereits im Wege der einstweiligen Anordnung etwas zugesprochen wird, was ihm erst über die Entscheidung in der Hauptsache gewährt werden kann. ${ }^{65}$ In einem Antragsverfahren nach $\S 123$ VwGO ist jedoch nur eine vorläufige Regelung möglich, die der Hauptsachenentscheidung noch Raum lässt. ${ }^{66}$ Hintergrund ist, dass ansonsten eine Verlagerung der gerichtlichen Klagen hin zu gerichtlichen Anträgen zu befürchten wäre, da die einstweiligen Anordnungen aufgrund der Eilbedürftigkeit schneller von den Gerichten entschieden werden, als die Klagen

Beachte: In Klausuren wird in der Regel eine Ausnahme zu diesem Grundsatz greifen!

Von dem Verbot der Vorwegnahme der Hauptsache sind aufgrund des Gebots

des effektiven Rechtsschutzes nach Art. 19 IV GG Ausnahmen anerkannt, wenn eine Versagung des vorläufigen Rechtsschutzes den Antragsteller schwer und unzumutbar oder irreparabel belasten würde. ${ }^{67}$ Dazu hat das BVerfG folgende Richtwerte getroffen:

BVerfG: ${ }^{68}$ „Je schwerer die mit einer Versagung von Eilrechtsschutz verbundenen Belastungen wiegen und je geringer die Wahrscheinlichkeit ist, dass sie im Falle des Obsiegens in der

63 Hummel, JuS 2011, 502 (503).

64 Das „Verbot der Vorwegnahme der Hauptsache“ ist nicht unumstritten. Es gibt zahlreiche Stimmen in der Literatur, die dieses ablehnen oder zumindest kritisieren. So z. B. Kuhla, in: Posser/Wolff, VwGO, 33. Ed., Stand: 1.4.2015, § 123 Rn. 154. Weitgehende Einigkeit besteht hingegen darin, dass eine endgültige Vorwegnahme der Hauptsache verboten sein soll. S. zu diesem Streitstand die vertiefenden Ausführungen von Hong, NVwZ 2012, 468.

65 Sodan/Ziekow, Grundkurs Öffentliches Recht, 8. Aufl. 2018, § 107 Rn. 9.

66 Kuhla, in: Posser/Wolff, VwGO, 50. Ed., Stand: 1.7.2019, § 123 Rn. 147.

67 Hufen, Verwaltungsprozessrecht, 11. Aufl. 2019, § 33 Rn. 18.

68 BVerfG, Beschl. v. 6.7.2016, Az.: 1 BvR 1705/15 = NJW 2017, 545.

Mira Wichmann 
Hauptsache rückgängig gemacht werden können, umso weniger darf das Interesse an einer vorläufigen Entscheidung zurückgestellt werden.“

BVerfG: ${ }^{69}$ „Der vorläufige Rechtsschutz ist also zu gewähren, wenn sonst dem Antragsteller eine erhebliche, über Randbereiche hinausgehende Verletzung in seinen Rechten droht, die durch die Entscheidung in der Hauptsache nicht mehr beseitigt werden kann, es sei denn, dass ausnahmsweise überwiegende, besonders gewichtige Gründe entgegenstehen.“

Das BVerfG spricht dem Anordnungsgrund in diesen Fällen ein solches Gewicht zu, sodass dem Antragsteller ein weiteres Zuwarten nicht zugemutet werden kann. Ein effektiver Rechtsschutz könnte dann nicht mehr gewährt werden. Zusammengefasst müssen also unzumutbare Nachteile zu befürchten sein, die über die typischen Belastungen des reinen Zeitverlusts hinausgehen und somit die Dringlichkeit der erstrebten einstweiligen Anordnung rechtfertigen. ${ }^{70}$

Neben dem grundsätzlichen Verbot der Vorwegnahme der Hauptsache kann das Gericht auch hinsichtlich seines Entscheidungsumfangs begrenzt sein. Nur, was in der Hauptsache geregelt werden kann, kann auch bereits in dem vorgeschalteten einstweiligen Rechtsschutz erreicht werden. Kurz gesagt, darf im Antragsverfahren nicht mehr als in der Hauptsache gewährt werden. ${ }^{71}$ Hat das Gericht beispielsweise über einen Anordnungsanspruch zu entscheiden, dessen Ausgestaltung im Beurteilungs- und Ermessensspielraum der Behörde steht, darf es nicht zu einer sog. Überschreitung der Hauptsache kommen. Eine solche Überschreitung liegt vor, wenn das Gericht der Hauptsache nur ein Bescheidungsurteil erlassen könnte, im Rahmen der einstweiligen Anordnung aber bereits die begehrte Begünstigung zuspricht.

Eine solche Überschreitung ist somit nur zulässig, wenn sich innerhalb des einstweiligen Verfahrens keine eindeutigen Beschränkungen des Ermessensspielraumes ergeben und es ansonsten zu einer irreversiblen Vereitelung der Grundrechtsposition oder einem schwerwiegenden Rechtsnachteil für den Antragsteller kommen würde. ${ }^{72}$

\section{Literaturhinweise}

Aufsätze: Hummel, Der vorläufige Rechtsschutz im Verwaltungsprozess (3. Teil), JuS 2011, 502ff.; Buchheister, Vorläufiger Rechtsschutz nach der VwGO aus richterlicher Sicht - Das Eilverfahren als Ersatz für das Hauptsacheverfahren?, DVBl

69 BVerfG, Beschl. v. 28.9.2009, Az.: 1 BvR 1702/09 = BeckRS 2009, 39313.

70 Kuhla, in: Posser/Wolff, VwGO, 49. Ed., Stand: 1.7.2018, § 123 Rn. 156.

71 Gersdorf, Verwaltungsprozessrecht, 6. Aufl. 2019, Rn. $215 \mathrm{ff}$.

72 Kuhla, in: Posser/Wolff, VwGO, 50. Ed., Stand: 1.7.2019, § 123 Rn. 158; Gersdorf, Verwaltungsprozessrecht, 6. Aufl. 2019, Rn. 218. 
2017, 610 ff; Guckelberger, Zulässigkeit und Anfechtbarkeit von verwaltungsgerichtlichen Hängebeschlüssen, NVwZ 2001, 275 ff.; Mann, Der verwaltungsrechtliche Hängebeschluss - ein schwarzes Loch des Verwaltungsprozessrechts, NWVbl. 2017, 60 ff.; Schenke, Verfassungsgerichtliche Verwerfungsmonopole und verwaltungsgerichtlicher vorläufiger Rechtsschutz, JuS 2017, 1141.

Fälle Fortgeschrittene: Fall 15 in: Eisentraut, Fälle zum Verwaltungsrecht, 2020; Helbich/Schübler-Pfister, Zirkus um den Zirkus, JuS 2017, 521; Blenk/ Schultes, Übungshausarbeit: Familiennachzug und Unionsbürgerschaft (Teil 1), ZAR 2018, 345.

Aktenvortrag: Weber, Kein Platz für Kinder, JA 2014, 460

\section{Der Antrag nach $\S 123$ VwGO im Baurecht (Felix Steengrafe)}

Grundsätzlich ist ein Antrag nach $\S 123$ VwGO auf eine einstweilige Anordnung 45 auch im Baurecht, etwa auf Erlass einer bauordnungsrechtlichen Verfügung, denkbar.

Beispiele sind Anträge eines Nachbarn auf einstweiligen Erlass einer Einstellungsverfügung (s. dazu näher \& 2 Rn. 1247ff.) oder einer Nutzungsuntersagung (s. dazu näher $§ 2$ Rn. 1243ff.).

Aufgrund des Verbotes der Vorwegnahme der Hauptsache ist eine Beseitigungsanordnung ausgeschlossen. ${ }^{73}$ Der Antrag kann auch auf die Anordnung geeigneter Maßnahmen zur Reduzierung des Baustellenlärmes gerichtet sein. ${ }^{74}$

\section{Der Antrag nach § 123 VwGO im Kommunalrecht (Sebastian Piecha)}

Im einstweiligen Rechtsschutzverfahren nach $\S 123$ VwGO im Kommunalrecht 46 gelten die allgemeinen Vorschriften (s. Rn. 1ff.); Besonderheiten ergeben sich nicht.

Bedeutende Weichenstellung ist, wie zuvor schon erläutert (s. § 2 Rn. 1271ff.), 47 insbesondere die Abgrenzung, ob es sich bei der angegriffenen Maßnahme der Kommune oder Aufsichtsbehörde um einen Verwaltungsakt im Sinne von $\S 35$ S. 1 VwVfG handelt oder nicht und welche Klageart im Hauptsacheverfahren

73 OVG Berlin-Brandenburg, Beschl.v. 14.10.2009, Az.: 2 S 54/09; Mehde/Hansen, NVwZ 2010, 14 (17).

74 VGH Mannheim, Beschl.v. 5.2.2015, Az.: 10 S 2471/14.

Mira Wichmann/Felix Steengrafe/Sebastian Piecha 
statthaft wäre, da sich hiernach die Statthaftigkeit des Verfahrens nach $\S 80 \mathrm{~V}$ VwGO bzw. § 123 VwGO bemisst.

48 Kommunalrechtlich sind hier insbesondere Sachverhalte denkbar, die etwa die Zulässigkeit von Bürgerbegehren, die Aufhebung von Ratsbeschlüssen oder die Untersagung oder Rücknahme bestimmter Äußerungen etwa des Bürgermeisters ${ }^{75}$ betreffen. Auch spielen Zulassungen zu öffentlichen Einrichtungen (Parteitag in Stadthalle) oft eine Rolle in Eilsachen.

75 Beispielfall zu Rechtsfragen kommunaler Satzungen s. Funke/Papp, JuS 2010, 395 ff. 